\title{
Genotypic diversity, antimicrobial resistance and biofilm-forming abilities of Campylobacter isolated from chicken in Central China
}

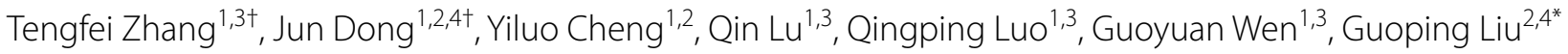
and Huabin Shao $1,2,3^{*}$

\begin{abstract}
Background: Campylobacter is considered to be the leading cause of human bacterial gastroenteritis, of which poultry is the main reservoir. Campylobacter contaminated chicken products are a major cause of human Campylobacter infection. In this study, the prevalence of Campylobacter in chicken in central China was investigated, and the genotypic diversity, antimicrobial resistance and biofilm of these isolates were characterized.

Results: A total of 206 Campylobacter isolates, including 166 C. jejuni and 40 C. coli, were isolated from chicken farms and live poultry markets in central China. Multilocus sequence typing and phylogenetic analysis showed that the Campylobacter isolates had diverse genetic backgrounds, which covered most of the dominant clone complexes (CCs) reported throughout China. The most prevalent CCs were CC-464, CC-1150, CC-353, and CC-828. All the isolates showed resistance to norfloxacin, ciprofloxacin and Cefazolin, and a prevalent resistance to fluoroquinolones, $\beta$-lactams and tetracyclines was also observed. Among all the isolates, 133 strains showed the ability to form biofilm, thereinto, the isolates in two genetic branches, mainly including CC-21, CC-48, CC-677 and CC-45, showed a significantly lower ability to form biofilm than other genetic branches $(p<0.05)$. However, in general, the ability to form biofilm varied among different genetic branches, suggesting a complex genetic background to biofilm formation, but not only the genetic lineages. Compared with the strains unable to form biofilm, biofilm-producing strains possessed a significantly higher resistance to ampicillin, neomycin, sulfamethoxazole, amikacin, clindamycin and erythromycin $(p<0.05)$.

Conclusions: To the best of our knowledge, this is the first report on the relationship of the genotypic diversity, antimicrobial resistance and biofilm-forming abilities of Campylobacter isolated from chicken in Central China, which showed the potential importance of biofilm in antimicrobial resistance. This study will help us better understand the epidemiology and antimicrobial resistance of Campylobacter.
\end{abstract}

Keywords: Campylobacter, Prevalence, Antibiotic resistance, Genotype lineage, Biofilm

\footnotetext{
*Correspondence: hhaaiieerr@163.com; shhb1961@163.com

†Tengfei Zhang and Jun Dong authors contributed equally to this work

${ }^{1}$ Key Laboratory of Prevention and Control Agents for Animal Bacteriosis,

Institute of Animal Husbandry and Veterinary, Hubei Academy

of Agricultural Sciences, Wuhan, China

${ }^{2}$ College of Animal Science, Yangtze University, Jingzhou, China

Full list of author information is available at the end of the article
} 


\section{Background}

Campylobacter is considered to be the leading cause of human bacterial gastroenteritis worldwide [1], accounting for an estimated 500 million infections per year globally [2]. In severe cases of $C$. jejuni infection, individuals may develop post infection complications associated with Guillain Barré Syndrome [3]. In North China, 36 cases of Guillain Barré Syndrome, resulted from C. jejuni infection, were reported in 2007 [4].

Campylobacter species, mainly including C. jejuni and C. coli, widely colonize in the intestinal tract of wild and domesticated animals and birds [5-7]. Chicken is one of the most popular animal-based food sources worldwide, which is also the reservoir of Campylobacter. Campylobacter-contaminated chicken products are a major cause of human Campylobacter infection [8], which highlights its potential public health threat. Several epidemiologic studies on Campylobacter have been carried out in some parts of China. From 2008 to 2014, Wang et al. reported that the positive rates of $C$. jejuni and $C$. coli were 18.1 and $19.0 \%$ respectively in five provinces of China [9]. Zhang et al. analyzed the genetic diversity of the $C$. jejuni isolates in Eastern China by multilocus sequence typing (MLST) and identified 94 sequence types (STs) belonging to 18 clonal complexes (CCs) [10]. However, data on the prevalence and genetic diversity of Campylobacter is still limited in China, especially central China, which is an important transportation junction.

Moreover, Campylobacter isolates have raised great concerns due to a frequent emergence of resistance to fluoroquinolone, erythromycin, and other drugs [11, 12], which limits treatment alternatives. Therefore, analysis of antimicrobial resistance of Campylobacter in the poultry industry will contribute to managing cognate infections and mitigating the emergence of antimicrobial resistant strains. Recent years, the multidrug-resistant Campylobacter have been frequently isolated, and a high antimicrobial resistance rate of Campylobacter, especially to fluoroquinolone, has been reported in many areas [13-15]. Multi-drug resistance of Campylobacter is more severe in China where the resistance to fluoroquinolones was reported to be as high as $98 \%$ in some areas $[16,17]$. Although some of the mechanisms accounting for antimicrobial resistance in Campylobacter have been revealed [11, 18, 19], some possible factors may also attribute to the raise of antimicrobial resistance, such as the ability of biofilm formation.

Our previous study has shown that the Campylobacter positive rate was $17.2 \%$, with bacterial count varying from 3.6 to 360 most-probable-number (MPN)/g in the positive samples of chicken meats collected from markets in central China [20]. Studying the prevalence of Campylobacter in live chicken and their surroundings will help us further control these pathogens. In this study, we investigated the prevalence, antimicrobial resistance and genetic diversity of Campylobacter strains isolated from chicken farms and markets in central China, which is one of the most important livestock and poultry circulation centers. We also tested the biofilm-forming ability of the Campylobacter isolates and analyzed the potential correlation among biofilm formation, genotypes, and antimicrobial resistance.

\section{Methods \\ Sampling and isolation of Campylobacter}

From 2012 to 2016, a total of 817 samples, including 317 anal swabs, 15 soils and 12 aerosols collected from chicken farms, and 448 anal swabs, 15 soils and 10 aerosols collected from live poultry markets, were collected in central China (3 farms and 4 markets in Hubei, 2 farms and 3 markets in Henan, 2 farms and 2 markets in Jiangxi and 1 farm and 2 markets in Anhui). In each sampling site, 35-45 anal swabs were collected. In parts of sampling sites, 2 aerosol samples and 3 soil samples were collected. Freshly collected anal swabs or soils were kept into Cary-Blair modified transport media (AMRESCO, USA). Aerosols (375 L/sample) were collected using BioSamper (SKC Ltd, USA). The samples were transported to the laboratory for Campylobacter isolation. The samples were resuspended in PBS which were used to inoculate Bolton broth containing Campylobacter growth and selective supplements (Oxoid, England) and incubated at $42{ }^{\circ} \mathrm{C}$ for $24 \mathrm{~h}$ in air tight jars containing the AnaeroPack (Mitsubishi, Japan) to generate a microaerobic condition. $100 \mu \mathrm{l}$ of the culture was spread onto a modified charcoal cefoperazone deoxycholate agar (mCCDA, Oxoid) plate containing Campylobacter selective supplements and incubated for $48 \mathrm{~h}$ at $42{ }^{\circ} \mathrm{C}$ under microaerobic condition [21]. The suspected Campylobacter colonies were further purified and identified by PCR as described [22]. C. jejuni and $C$. coli were differentiated by hippuric acid hydrolysis test and PCR test [6]. The identified Campylobacter strains were stored at $-80{ }^{\circ} \mathrm{C}$ in $\mathrm{MH}$ broth containing 30\% (v/v) glycerol.

\section{Antibiotic resistance profiles}

The antibiotic susceptibility of the isolates was determined by the disk diffusion method on Mueller-Hinton Agar (MHA, Oxoid) according to the Clinical and Laboratory Standards Institute Standards guidelines [23]. A total of 11 antibiotics were tested, including ampicillin (Amp, $10 \mu \mathrm{g}$ ), Ceftriaxone (Cet, $30 \mu \mathrm{g}$ ), Cefazolin (Cez, $30 \mu \mathrm{g}$ ), amikacin (Ami, $30 \mu \mathrm{g})$, Neomycin (Neo, $30 \mu \mathrm{g})$, tetracycline (Tet, $10 \mu \mathrm{g}$ ), sulfamethoxazole (Sul, $300 \mu \mathrm{g}$ ), clindamycin (Cli, $10 \mu \mathrm{g})$, erythromycin (Ery, $10 \mu \mathrm{g})$, ciprofloxacin (Cip, $5 \mu \mathrm{g}$ ) and norfloxacin (Nor, $10 \mu \mathrm{g}$ ). After 
incubation for $24 \mathrm{~h}$ at $37^{\circ} \mathrm{C}$, the diameters (in mm) of the inhibition zones were measured. E. coli strain ATCC 25922 and C. jejuni strain ATCC 33560 were used as the quality control.

\section{Biofilm assays}

Biofilm formation was assessed as described [24]. Briefly, overnight cultured cells were adjusted to an $\mathrm{OD}_{590 \mathrm{~nm}}$ of 0.1 in Brucella medium (Oxoid) supplemented with 5\% $(\mathrm{v} / \mathrm{v})$ chicken juice. To allow biofilm formation, $1 \mathrm{ml}$ of the cell culture was added to a 24-well polystyrene tissue culture plate (Corning) which was incubated at $37^{\circ} \mathrm{C}$ under microaerobic condition for $48 \mathrm{~h}$ before staining. For crystal violet staining, cells were discarded and each well was washed with water, and the plate was then dried at $60{ }^{\circ} \mathrm{C}$ for $30 \mathrm{~min}$. One milliliter of $1 \%$ crystal violet solution was added to each well, and the plate was incubated on a rocker at room temperature for $30 \mathrm{~min}$. Unbound crystal violet was washed off with water and the plate was dried at $37{ }^{\circ} \mathrm{C}$. Bound crystal violet was dissolved in $20 \%(\mathrm{v} / \mathrm{v})$ acetone-containing ethanol for $10 \mathrm{~min}$. The dissolved crystal violet was then poured into cuvettes and $\mathrm{OD}_{630 \mathrm{~nm}}$ was measured. All the tests were performed in triplicate. Three wells were subject to the same treatment but without bacteria inoculated, which were used as the negative control. The cutoff OD value (ODc) was defined as two times of the negative control value as previously reported $[25,26]$. Based on the OD values, strains were classified into the following three categories: non-biofilm producer (OD $\leq \mathrm{ODc}$ ), weak biofilm producer (ODc $<\mathrm{OD} \leq 2 \times \mathrm{ODc}$ ) and strong biofilm producer $(2 \times$ ODc $<$ OD) .

\section{MLST typing}

In order to determine the genetic diversity of the Campylobacter isolates and their relationship, MLST analysis was carried out as previously described [27]. Briefly, genomic DNA was extracted using MiniBEST Universal Genomic DNA Extraction Kit (TaKaRa, Dalian, China) according to the manufacturer's instructions. MLST analysis was conducted by sequencing seven Campylobacter housekeeping genes (aspA, gln $A, g l t A, g l y A, p g m$, $t k t$, and $u n c A$ ). The primers sets for these seven genes and their amplification conditions were used as previously described [27]. Amplification products were purified and sequenced. Allele numbers, sequence types (STs) and clonal complexes (CCs) were assigned using the Campylobacter MLST database (http://pubmlst.org/ Campylobacter/). Novel STs were submitted to MLST database and assigned new numbers. Consensus network of the calculated tree was constructed by SliptsTree 4 version 1.2 using the multi-aligned core genome sequence of the different STs.

\section{Statistical analysis}

Chi square was used to determine the significance of resistance rates in different biofilm-forming groups and to compare the isolation rates of $C$. jejuni and C. coli. For analyzing the biofilm-forming abilities, the mean OD values (mean \pm SEM) of isolates in each clade were calculated, and two-tailed $t$ tests were used to determine the significance of the biofilm-forming abilities in different clades $p<0.05$ was considered statistically significant.

\section{Results}

\section{Prevalence of Campylobacter}

As shown in Table 1, 206 Campylobacter strains (positive rate 25.2\%) were isolated, including 156 C. jejuni and 38 C. coli from anal swab samples, 5 C. jejuni and 2 C. coli from soil samples, and $5 C$. jejuni from aerosol samples. The isolation rate of $C$. jejuni was higher than that of $C$. coli $(20.3 \%$ vs $4.9 \%, p=0.000)$. Among these isolates, 66

Table 1 The prevalence of Campylobacter in chicken farms and poultry markets in central China

\begin{tabular}{|c|c|c|c|c|c|c|c|c|c|}
\hline \multirow[t]{3}{*}{ Sources } & \multirow[t]{3}{*}{ Sampling site (no.) } & \multicolumn{8}{|c|}{ No. of positive samples/collected samples } \\
\hline & & \multicolumn{2}{|c|}{ Anal swabs } & \multicolumn{2}{|l|}{ Soils } & \multicolumn{2}{|l|}{ Aerosols } & \multicolumn{2}{|l|}{ Total } \\
\hline & & C. jejuni & C. coli & C. jejuni & C. coli & C. jejuni & C. coli & C. jejuni & C. coli \\
\hline \multirow[t]{4}{*}{ Chicken farms } & Hubei (3) & $23 / 112$ & $8 / 112$ & $1 / 9$ & $0 / 9$ & $4 / 8$ & $0 / 8$ & $66 / 344$ & $19 / 344$ \\
\hline & Henan (2) & $12 / 83$ & $5 / 83$ & $1 / 6$ & $1 / 6$ & $1 / 4$ & $0 / 4$ & & \\
\hline & Anhui (2) & $15 / 82$ & $4 / 82$ & N/A & N/A & N/A & N/A & & \\
\hline & Jiangxi (1) & $9 / 40$ & $1 / 40$ & N/A & N/A & N/A & N/A & & \\
\hline \multirow[t]{4}{*}{ Poultry markets } & Hubei (4) & $32 / 167$ & $9 / 167$ & $1 / 9$ & $1 / 9$ & $0 / 6$ & $0 / 6$ & $100 / 473$ & $21 / 473$ \\
\hline & Henan (3) & $31 / 117$ & $4 / 117$ & $2 / 6$ & $0 / 6$ & $0 / 4$ & $0 / 4$ & & \\
\hline & Anhui (2) & $19 / 86$ & $4 / 86$ & N/A & N/A & N/A & N/A & & \\
\hline & Jiangxi (2) & $15 / 78$ & $3 / 78$ & N/A & N/A & N/A & N/A & & \\
\hline Total & & $156 / 765$ & $38 / 765$ & $5 / 30$ & $2 / 30$ & $5 / 22$ & $0 / 22$ & $166 / 817$ & $40 / 817$ \\
\hline
\end{tabular}


C. jejuni (positive rate 19.2\%) and 19 C. coli (positive rate $5.5 \%$ ) were isolated from the 344 samples collected from poultry farms, and $100 \mathrm{C}$. jejuni (positive rate $21.1 \%$ ) and 21 C. coli (positive rate 4.4\%) were isolated from the 473 samples collected from live poultry markets.

\section{Genetic diversity of Campylobacter}

MLST was performed to determine the genetic diversity and clonal origins of the Campylobacter isolates, and the details of MLST results have been listed in Additional file 1: Table S1. As shown in Fig. 1, 206 isolates contained a total of 72 different STs in our test. Among these isolates, 146 out of the 206 isolates possessed 50 different STs which belonged to $15 \mathrm{CCs}$. The remaining 60 isolates belonged to 22 different unassigned STs. 40 novel STs harboring 106 isolates were isolated for the first time. CC-464 was the most frequently isolated clonal complex which contained 33 isolates belonging to ten STs, and accounted for $16.0 \%(33 / 206)$ of all the isolates. The major clonal complexes also included CC-1150 $(\mathrm{n}=25$, $12.1 \%), \mathrm{CC}-353(\mathrm{n}=22,10.7 \%)$ and CC-828 $(\mathrm{n}=16$, $7.8 \%$ ). The isolates collected from markets covered $61 \mathrm{STs}$ belonging to $12 \mathrm{CCs}$ and unassigned, while those isolated from chicken farms harbored 45 STs belonging to 12 CCs and unassigned.

All the STs were classified into five major clades (Fig. 1). Clade 1 covered most of the isolates, including the two major clonal complexes CC-464 and CC-353. All of the C. coli isolates belonging to CC-1150 and CC-828 were clustered in clade 2 . The isolates recovered from chicken markets and farms shared nine out of the fifteen clonal complexes and all the clades contained isolates recovered from chicken farms and markets, suggesting that the isolates collected from the two places might share same origins.

\section{Antimicrobial resistance profiles of Campylobacter}

As shown in Fig. 2a, all of the C. jejuni and C. coli isolates showed resistance to norfloxacin, ciprofloxacin and Cefazolin. A high rate of resistance to tetracycline, ceftriaxone and ampicillin has also been observed for the isolates, among which $89.69 \%$ of the C. jejuni and $90.24 \%$ of the $C$. coli were resistant to tetracycline; $82.42 \%$ of the C. jejuni and $97.56 \%$ of the C. coli were resistant to ceftriaxone; and $76.36 .5 \%$ of the C. jejuni and $82.93 \%$ of the C. coli were resistant to ampicillin. The isolates showed a relative low rate of resistance to amikacin $(4.85 \%$ of the C. jejuni and $21.95 \%$ of the C. coli), neomycin $(10.91 \%$ of the $C$. jejuni and $14.63 \%$ of the C. coli), and erythromycin (12.12\% of the C. jejuni and $26.83 \%$ of the C. coli). Except for sulfamethoxazole and the three drugs which all the isolates were resistant to, the resistance rates of $C$. jejuni were lower than that of $C$. coli in this study.
As shown in Fig. 2b, all the isolates were resistant to at least three tested antimicrobial agents, among which $95.1 \%$ of the isolates were resistant to more than five antibiotics. There were four $C$. jejuni and two C. coli strains that were resistant to all the eleven antimicrobial agents tested. However, one C. jejuni strain showed sensitivity to most of the antibiotics, which was only resistant to three of antimicrobial agents tested. In general, most of the isolates were resistant to 5-7 antimicrobial agents tested. The most frequent multidrug resistance pattern was resistance to ciprofloxacin, norfloxacin, tetracycline, ampicillin, ceftriaxone and cefazolin, which covered 54 isolates (Additional file 1: Table S1).

\section{Biofilm formation of Campylobacter isolates}

The cutoff $\mathrm{OD}$ value (ODc) to define a biofilm producer was determined as $\mathrm{OD}_{630 \mathrm{~nm}}=0.279$ as previously described [26]. The $\mathrm{OD}_{630 \mathrm{~nm}}$ values generated by crystal violet staining of each isolate were listed in Additional file 1: Table S1. Based on the biofilm-forming ability, 206 Campylobacter isolates were classified to three groups (Table 2, Fig. 3). Seventy-three isolates (35.4\%) were identified as non-biofilm producers $\left(\mathrm{OD}_{630} \leq 0.279\right)$, while 133 isolates (64.6\%) were biofilm producers. Among these biofilm producers, 113 isolates were weak biofilm producers $\left(0.279<\mathrm{OD}_{630} \leq 0.558\right)$ and 20 isolates were strong biofilm producers $\left(\mathrm{OD}_{630}>0.558\right)$. All the soil isolates and aerosol isolates were biofilm producers.

\section{Correlation between biofilm formation and antimicrobial resistance}

As shown in Table 2, both biofilm producers and nonbiofilm producers showed a high rate of resistance to ceftriaxone, cefazolin, tetracycline and two fluoroquinolones drugs including ciprofloxacin and norfloxacin. However, compared with non-biofilm producers, biofilm producers possessed a higher rate of resistance to ampicillin $(88.0 \%$ vs $58.9 \%, p=0.000)$, neomycin $(18.0 \%$ vs $2.7 \%, p=0.002)$ and sulfamethoxazole $(48.9 \%$ vs $8.2 \%$, $p=0.000$ ). Moreover, all of the non-biofilm producers were sensitive to amikacin, clindamycin and erythromycin. In contrast, strong biofilm producers showed a resistance rate of more than $90 \%$ to clindamycin and erythromycin. Six isolates, which were resistant to all the types of antibiotics tested, were biofilm producers. Among them, four out of the six isolates were strong biofilm producers (Additional file 1: Table S1). These results suggested that the ability of biofilm formation had a positive correlation with antimicrobial resistance of the Campylobacter isolates. However, there was an exception that compared with the non-biofilm producers, the biofilm producers showed a lower rate of resistance to tetracycline. 


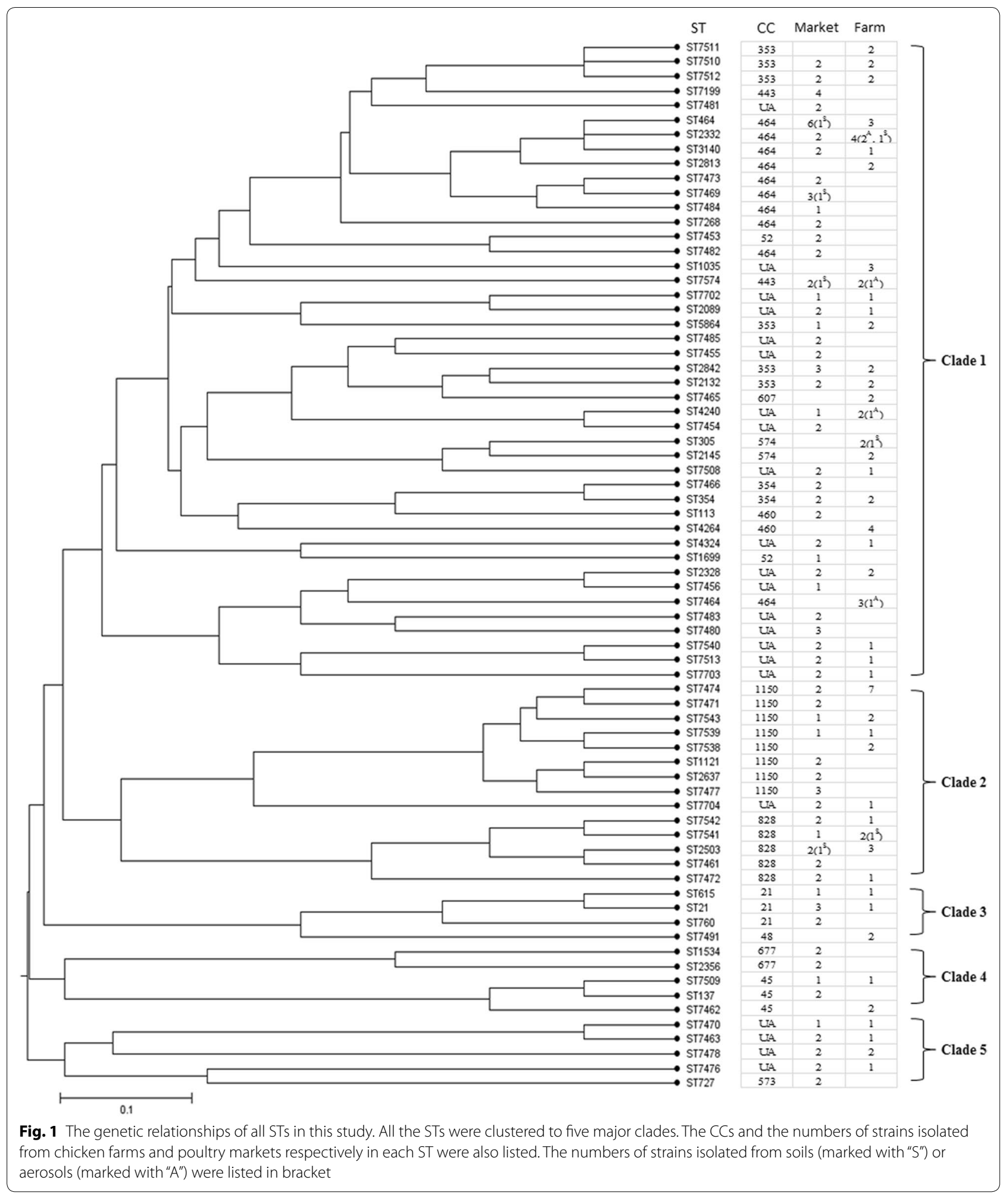



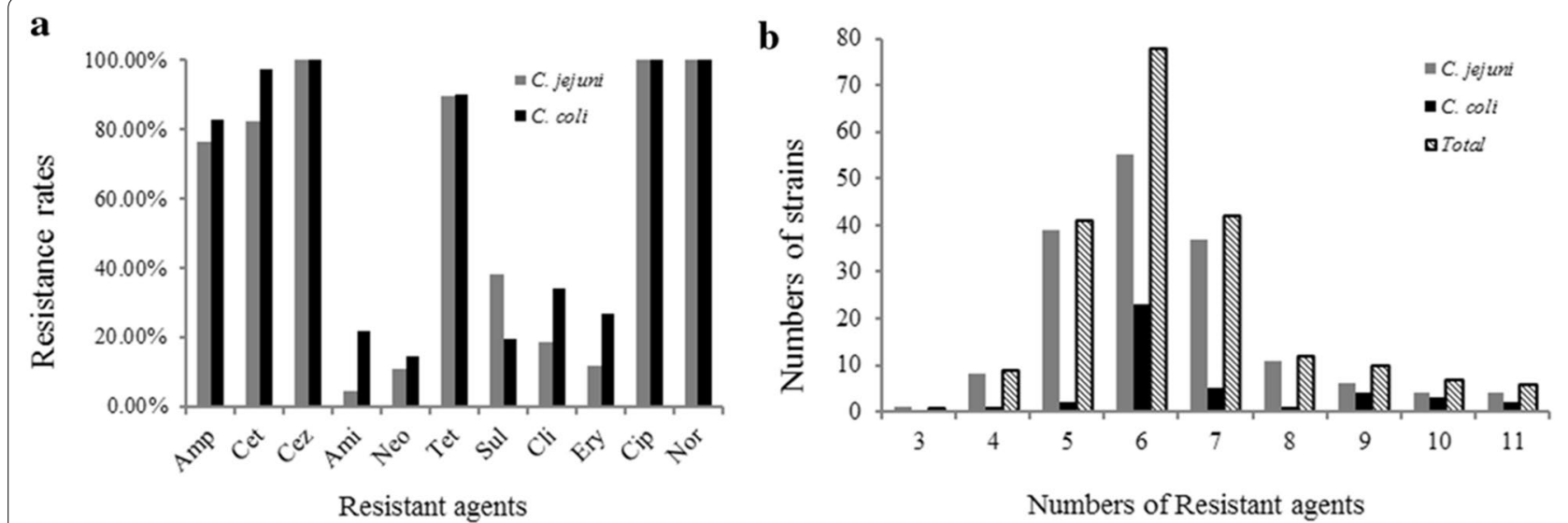

Fig. 2 Antimicrobial resistance of Campylobacter isolates. a Resistance rates of C. jejuni and C. coli isolates to 11 agents; $\mathbf{b}$ multidrug resistance of $C$. jejuni and C. coli isolates to 11 agents

Table 2 Antimicrobial resistance of Campylobacter isolates with different biofilm-forming abilities

\begin{tabular}{|c|c|c|c|c|c|c|c|}
\hline \multirow[t]{2}{*}{ Classes } & \multirow[t]{2}{*}{ Members } & \multicolumn{2}{|c|}{ Biofilm strong isolates $(n=20)$} & \multicolumn{2}{|c|}{ Biofilm weak isolates $(n=113)$} & \multicolumn{2}{|c|}{ Biofilm negative isolates $(n=73)$} \\
\hline & & $\begin{array}{l}\text { No. of resistant } \\
\text { isolates }\end{array}$ & $\begin{array}{l}\text { Resistance rates } \\
\text { (\%) }\end{array}$ & $\begin{array}{l}\text { No. of resistant } \\
\text { isolates }\end{array}$ & $\begin{array}{l}\text { Resistance } \\
\text { rates (\%) }\end{array}$ & $\begin{array}{l}\text { No. of resistant } \\
\text { isolates }\end{array}$ & $\begin{array}{l}\text { Resistance rates } \\
\text { (\%) }\end{array}$ \\
\hline \multirow[t]{3}{*}{$\beta$-lactams } & Ampicillin & 19 & 100 & 98 & 85.8 & 43 & 58.9 \\
\hline & Ceftriaxone & 19 & 95.0 & 97 & 85.8 & 60 & 82.2 \\
\hline & Cefazolin & 20 & 100 & 113 & 100.0 & 73 & 100.0 \\
\hline \multirow[t]{2}{*}{ Aminoglycosides } & Neomycin & 11 & 55.0 & 13 & 11.5 & 2 & 2.7 \\
\hline & Amikacin & 8 & 40.0 & 9 & 8.0 & 0 & 0 \\
\hline Tetracyclines & Tetracycline & 16 & 80.0 & 99 & 84.1 & 70 & 95.9 \\
\hline Sulfonamides & $\begin{array}{l}\text { Sulfamethoxa- } \\
\text { zole }\end{array}$ & 19 & 95.0 & 46 & 40.7 & 6 & 8.2 \\
\hline \multirow[t]{2}{*}{ Fluoroquinones } & Ciprofloxacin & 20 & 100 & 113 & 100.0 & 73 & 100.0 \\
\hline & Norfloxacin & 20 & 100 & 113 & 100.0 & 73 & 100.0 \\
\hline Lincosamides & Clindamycin & 19 & 95.0 & 26 & 23.0 & 0 & 0 \\
\hline Macrolides & Erythromycin & 18 & 90.0 & 13 & 11.5 & 0 & 0 \\
\hline
\end{tabular}

\section{Correlation between biofilm formation and genotypes}

As shown in Figs. 1 and 3, 206 Campylobacter isolates fell into five clades, of which clade 1 and 2 had more members than the other clades. The mean OD values in each clade were as follows: clade $1=0.37 \pm 0.01$, clade $2=0.37 \pm 0.02$, clade $3=0.25 \pm 0.04$, clade $4=0.26 \pm 0.03$ and clade $5=0.31 \pm 0.03$. Although all of the strong biofilm producers were present in clade 1 and clade 2, strains in these two clades exhibited different levels of abilities to form biofilm, which suggested that the ability of biofilm formation varied among the dominant genotypes of Campylobacter. $75 \%$ of the strains (20/25) in clade 3 and clade 4 were non-biofilm producers and the ability of biofilm-formation of the strains in these two clades was significantly lower than that of the other clades (clade 3 vs clade $1, p=0.007$; clade 3 vs clade $2, p=0.013$; clade 4 vs clade $1, p=0.012$; clade 4 vs clade $2, p=0.020)$. Strains which belonged to CC-21, CC-48, CC-677, CC-45 and a few unassigned isolates were included in these two clades.

\section{Discussion}

Poultry are recognized as a main reservoir of Campylobacter. Consumption of poultry is considered to be an important cause of human infection with Campylobacter, and leads to extensive spread antimicrobial resistance [28]. In this study, Campylobacter strains were isolated from $25.2 \%$ of the samples collected from chicken farms and markets, including $166 \mathrm{C}$. jejuni and 40 C. coli. According to several previous reports, the 


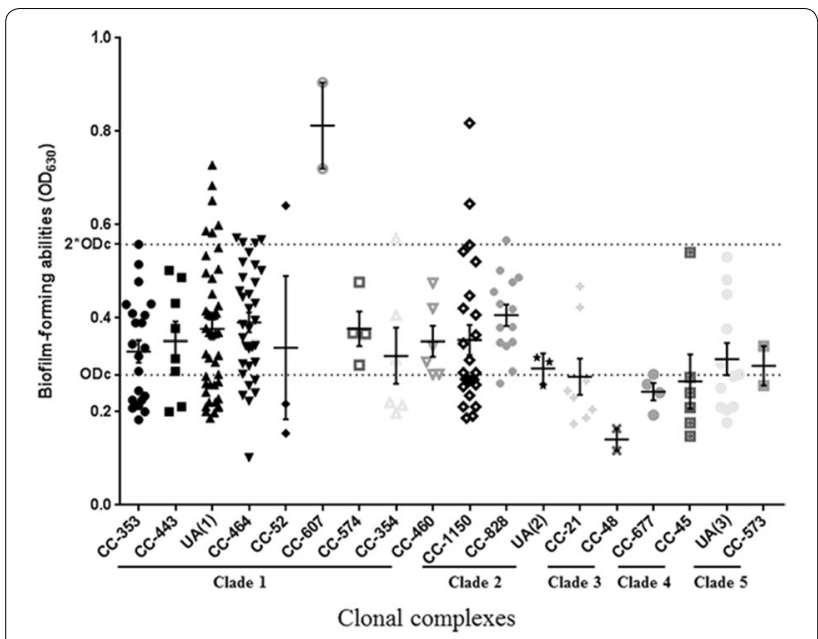

Fig. 3 Biofilm-forming abilities of Campylobacter isolates belonging to different clonal complexes. The bottom broken lines indicate the cutoff value $(O D C=0.279)$ and twofold cutoff value $(O D c=0.558)$. Based on the OD values, the strains were classified in three categories: non-biofilm producer (OD $\leq \mathrm{ODc})$, weak biofilm producer $(\mathrm{ODc}<\mathrm{OD} \leq 2 \times \mathrm{ODc})$ and strong biofilm producer $(2 \times \mathrm{ODc}<\mathrm{OD})$

positive detection rate of Campylobacter in poultry farms varies largely between different regions, ranging from 2 to $100 \%$, and the prevalence of Campylobacter is lower in Scandinavian countries than in other European countries, North America, and developing countries [29]. China is the biggest developing country in which a diverse prevalence rate has also been reported in different parts of the country. For example, Huang et al. revealed that $C$. jejuni was frequently detected in poultry, with an average isolation rate of up to $18.61 \%$ [30]. Wang et al. showed that the positive rates of $C$. jejuni and C. coli were 18.1 and $19.0 \%$ respectively in chicken in five provinces of China [9]. In Tianjin, the contamination rates of C. jejuni and other Campylobacter species were 13.7 and $5.7 \%$ respectively [31]. In this study, our data showed that the positive rate of $C$. jejuni was a bit higher than most of the other studies carried out in China. We also found that Campylobacter existed in the soils and aerosols of chicken farms and markets, suggesting that the pathogens were widely spreading between host and surroundings. This situation makes it harder for us to control Campylobacter infection. A prevalence and risk assessment of C. jejuni in chicken in China suggested that key efforts should be made, especially in chick breeding and chicken preparation processes [32].

In our study, MLST analysis showed a total of 72 different STs belonging to $15 \mathrm{CCs}$ and some unassigned clonal complexes. The major clonal complexes included CC-464, CC-1150, CC-353, and CC-828, which were similar to our previous investigation on chicken meat in the same region [21]. Most of these CCs (CC-464, CC-1150, CC-353 and CC-828) were also frequently identified in diarrhea patients worldwide [33, 34]. In North China, the most frequently isolated clonal complexes were CC-21, CC-353, CC-354 and CC-443 [31, 35], while the dominant clonal complexes of C. coli were CC-828 and CC-1150 [36]. In East China, the most common ST type of the Campylobacter strains isolated from human and food was ST-353, while the dominant ST type from chicken and food was ST-354 [10]. In Guangdong, a province in southern China, the dominant clonal complex was CC-828 [37]. It seems that the dominant clonal complexes of Campylobacter were discrepant in different regions. However, most of the CCs reported in these regions had been isolated in our study, which may be because central China, where all the samples were collected, is one of the most important livestock and poultry circulation centers in our country.

A total of 40 novel STs were identified in this study. Genetic relationship analysis showed that different sources of isolates have a crossed distribution in each clonal group and most of the novel STs only have a minor variation with a close phylogenetic relationship to known CCs. Selection forces, such as differences in temperature, structure and biochemical and immunological habitats, may accelerate the evolution to gain the ability to persist in different enteric environments and survive in different environments during transmission. Clade 1 and 2 contained lots of small genetic branches, which may be due to the adaptive evolution of isolates in these two clades occurred more frequently in our investigated regions.

Another more important selection pressure might be the usage of antibiotics, which could cause heritable genetic mutations and horizontal resistance gene transfer, leading to serious antimicrobial resistance in Campylobacter [11, 38]. More seriously, some of the antibiotics to which the Campylobacter isolates were resistant were used as therapeutic drugs in severe cases of infection [39]. Although resistance rates varied in different regions, in general high resistance rates, especially to fluoroquinolones, were found in most of the studies in China. For example, in Zhang et al.s study, the resistance rate of Campylobacter to ciprofloxacin was $100 \%$, and $94 \%$ to tetracycline, $61 \%$ to erythromycin, and $50 \%$ to ampicillin [36]. Chen et al. reported that more than $98 \%$ of the tested Campylobacter isolates were resistant to quinolones and tetracycline [40]. Even as early as in 2002, the prevalence of quinolone resistance of the isolates had been up to $85.9 \%$ in Hong Kong [41]. Low resistance rates of Campylobacter were only reported in Northwest China [42]. In our study, a very high resistance rate to $\beta$-lactams, tetracyclines and fluoroquinones was observed (Fig. 2), and a high resistance rate to the other 
drugs, such as erythromycin, was also found in the strong biofilm producers (Table 2). Our previous study showed that all of the fluoroquinolone-resistant strains contained a Thr-86-Ile substitution in GyrA, and that the CmeRBox variations increased the expression of CmeABC efflux pump which led to the high resistance [43]. Overexpression of drug efflux pump may not only contribute to fluoroquinolones resistance, but also increase resistance to other drugs [44-46]. Bacteria exposing in efflux inhibitors or mutants in efflux pumps showed decreased biofilm, which suggested that efflux pumps also contributed to their biofilm formation [47, 48]. Although more resistance mechanisms need to be revealed, efflux pumps seem to play important roles in antimicrobial resistance as well as biofilm formation.

Biofilms are sessile communities of bacterial cells enclosed in a self-produced extracellular polysaccharide matrix, which plays an important role in evading host immune clearance and resisting antimicrobial agents, leading to persistent and chronic infections [26]. Campylobacter may form a monospecies biofilm, which protects them from environmental stress, including antibiotic treatment [24]. In our tested strains, $64.6 \%$ were identified to be biofilm producers. Comparing with the non-biofilm producers, the biofilm producers possessed a higher resistance rate to ampicillin, neomycin, sulfamethoxazole, amikacin, clindamycin and erythromycin. Although studies on the correlation between biofilm and antimicrobial resistance were limited in Campylobacter, positive impact of biofilm on reducing the permeation of ampicillin has been reported in other bacteria [49]. Some regulators, such as LuxS, have also been reported to be linked to biofilm formation and antimicrobial resistance in some bacteria [50]. We found an exception that the resistance rate to tetracycline was higher in non-biofilm producing isolates than in biofilm producing strains, it may be due to the high distribution of the resistance genes in non-biofilm producing isolates, such as tet [51]. It is interesting that all of the soil isolates and aerosol isolates were biofilm producers, which suggested that biofilm might be an important factor to help strain to survive in the surroundings as well as in the host. Our study on the biofilm-forming characteristics of Campylobacter isolates would help us understand the increasing resistance to antibiotics of Campylobacter as well as their pathogenicity to host.

In clade 3 and clade $4,75 \%$ of the strains (20/25) were non-biofilm producers and the biofilm-forming abilities in these two clades were significantly lower than other clades $(p<0.05)$. The closely related strains may have a common ancestor, and STs developing from one biofilmforming ST origin may share better biofilm-forming ability. The correlation of the origin and phylogenetic relationship between their $C$. jejuni isolates and biofilmforming abilities has also been reported [52]. Previous studies also showed that some gene variants were associated with different $C$. jejuni multilocus sequence types, such as $f s p A$ [53] and cap $A$ [54]. The association between biofilm related genes and multilocus sequence types needs to be further studied. However, isolates within the same clade also exhibited varied abilities to form biofilm in our study. Ben et al. analyzed the genome sequences of strains with different biofilm-forming abilities, and found that three genes were associated with the increased biofilm formation in CC-21 and 43 genes in CC-45, but there was no overlap between these two CCs [55]. These results suggested a complex genetic correlation between genetic background and biofilm formation.

\section{Conclusions}

In the present study, a high prevalence and genotypic diversity were observed in the Campylobacter strains isolated in chicken in central China. We analyzed the correlation among biofilm-forming abilities, MLST genotype and antimicrobial resistance, which revealed a positive correlation between resistance rate and the ability of biofilm-forming. This study will help us better understand the epidemiology and resistance of Campylobacter.

\section{Additional file}

Additional file 1: Table S1. The details of each Campylobacter isolates.

\section{Abbreviations}

MLST: multilocus sequence typing; STs: sequence types; CCs: clonal complexes.

\section{Author' contributions}

The experiments were performed mainly by JD, TZ, YC and QL, and some experiments were performed with the help of QL and GW, TZ, JD and GL performed the data analysis. The study was designed by HS and GL. All authors read and approved the final manuscript.

\section{Author details \\ ${ }^{1}$ Key Laboratory of Prevention and Control Agents for Animal Bacteriosis, Institute of Animal Husbandry and Veterinary, Hubei Academy of Agricultural Sciences, Wuhan, China. ${ }^{2}$ College of Animal Science, Yangtze University, Jin- gzhou, China. ${ }^{3}$ Hubei Key Laboratory of Animal Embryo and Molecular Breed- ing, Institute of Animal and Veterinary Science, Hubei Academy of Agricultural Sciences, Wuhan, China. ${ }^{4}$ The Cooperative Innovation Center for Sustainable Pig Production, Wuhan, China.}

Acknowledgements

Not applicable.

Competing interests

The authors declare that they have no competing interests.

Availability of data and materials

The authors declare that all data supporting the findings of this study are available within the article. 


\section{Consent for publication}

Not applicable.

\section{Ethics approval and consent to participate}

The experiments were approved by the Ethics Committee of Hubei Academy of Agricultural Sciences according to Hubei Province Laboratory Animal Management Regulations-2005.

\section{Funding}

This study was supported by Chinese Key Research and Development Plan (2016YFD0500505), Chinese Special Fund for Agro-scientific Research in the Public Interest (201303044) and Chinese Agriculture Research System (CARS41-G13). CAAS Basic Research Business Expenses (Y2017PT50).

\section{Publisher's Note}

Springer Nature remains neutral with regard to jurisdictional claims in published maps and institutional affiliations.

Received: 11 September 2017 Accepted: 23 October 2017 Published online: 09 November 2017

\section{References}

1. Coker AO, Isokpehi RD, Thomas BN, Amisu KO, Obi CL. Human campylobacteriosis in developing countries. Emerg Infect Dis. 2002;8(3):237-44.

2. Epps SV, Harvey RB, Hume ME, Phillips TD, Anderson RC, Nisbet DJ. Foodborne Campylobacter: infections, metabolism, pathogenesis and reservoirs. Int J Environ Res Public Health. 2013;10(12):6292-304.

3. Goodfellow JA, Willison HJ. Guillain-Barre syndrome: a century of progress. Nat Rev Neurol. 2016;12(12):723-31.

4. Ye Y, Zhu D, Wang K, Wu J, Feng J, Ma D, Xing Y, Jiang X. Clinical and electrophysiological features of the 2007 Guillain-Barre syndrome epidemic in northeast China. Muscle Nerve. 2010;42(3):311-4.

5. Blaser MJ, LaForce FM, Wilson NA, Wang WL. Reservoirs for human campylobacteriosis. J Infect Dis. 1980;141(5):665-9.

6. Keller Jl, Shriver WG. Prevalence of three campylobacter species, C. jejuni, C. coli, and C. lari, using multilocus sequence typing in wild birds of the Mid-Atlantic region, USA. J Wildl Dis. 2014;50(1):31-41.

7. Weis AM, Miller WA, Byrne BA, Chouicha N, Boyce WM, Townsend AK. Prevalence and pathogenic potential of campylobacter isolates from free-living, human-commensal american crows. Appl Environ Microbiol. 2014;80(5):1639-44.

8. Horrocks SM, Anderson RC, Nisbet DJ, Ricke SC. Incidence and ecology of Campylobacter jejuni and coli in animals. Anaerobe. 2009;15(1-2):18-25.

9. Wang Y, Dong Y, Deng F, Liu D, Yao H, Zhang Q, Shen J, Liu Z, Gao Y, Wu C, Shen Z. Species shift and multidrug resistance of Campylobacter from chicken and swine, China, 2008-14. J Antimicrob Chemother. 2015;71(3):666-9.

10. Zhang G, Zhang X, Hu Y, Jiao XA, Huang J. Multilocus sequence types of Campylobacter jejuni isolates from different sources in eastern China. Curr Microbiol. 2015;71(3):341-6.

11. lovine NM. Resistance mechanisms in Campylobacter jejuni. Virulence. 2013;4(3):230-40.

12. Hao H, Dai M, Wang Y, Peng D, Liu Z, Yuan Z. 23 S rRNA mutation A2074C conferring high-level macrolide resistance and fitness cost in Campylobacter jejuni. Microb Drug Resist. 2009;15(4):239-44.

13. Klein-Jobstl D, Sofka D, Iwersen M, Drillich M, Hilbert F. Multilocus sequence typing and antimicrobial resistance of Campylobacter jejuni isolated from Dairy Calves in Austria. Front Microbiol. 2016;7:72.

14. Bakeli G, Sato K, Kumita W, Saito R, Ono E, Chida T, Okamura N. Antimicrobial susceptibility and mechanism of quinolone resistance in Campylobacter jejuni strains isolated from diarrheal patients in a hospital in Tokyo. J Infect Chemother. 2008;14(5):342-8.

15. Tang Y, Sahin O, Pavlovic N, LeJeune J, Carlson J, Wu Z, Dai L, Zhang Q. Rising fluoroquinolone resistance in Campylobacter isolated from feedlot cattle in the United States. Sci Rep. 2017;7(1):494.
16. Bai Y, Cui S, Xu X, Li F. Enumeration and characterization of Campylobacter species from retail chicken carcasses in Beijing, China. Foodborne Pathog Dis. 2014;11(11):861-7.

17. Nguyen TN, Hotzel H, El-Adawy H, Tran HT, Le MT, Tomaso H, Neubauer H, Hafez HM. Genotyping and antibiotic resistance of thermophilic Campylobacter isolated from chicken and pig meat in Vietnam. Gut Pathog. 2016;8:19.

18. Yao H, Liu D, Wang Y, Zhang Q, Shen Z. High prevalence and predominance of the aph ( $\left.2^{\prime \prime}\right)$-If gene conferring aminoglycoside resistance in Campylobacter. Antimicrob Agents Chemother. 2017;61(5):e00112-7.

19. Tang Y, Dai L, Sahin O, Wu Z, Liu M, Zhang Q. Emergence of a plasmidborne multidrug resistance gene $\mathrm{Cr}(\mathrm{C})$ in foodborne pathogen Campylobacter. J Antimicrob Chemother. 2017;72(6):1581-8.

20. Zhang T, Luo Q, Chen Y, Li T, Wen G, Zhang R, Luo L, Lu Q, Ai D, Wang H, et al. Molecular epidemiology, virulence determinants and antimicrobial resistance of Campylobacter spreading in retail chicken meat in Central China. Gut Pathog. 2016;8:48.

21. Zhang A, Song L, Liang H, Gu Y, Zhang C, Liu X, Zhang J, Zhang M. Molecular subtyping and erythromycin resistance of Campylobacter in China. J Appl Microbiol. 2016;121(1):287-93.

22. Tang JY, Nishibuchi M, Nakaguchi Y, Ghazali FM, Saleha AA, Son R. Transfer of Campylobacter jejuni from raw to cooked chicken via wood and plastic cutting boards. Lett Appl Microbiol. 2011;52(6):581-8.

23. Serichantalergs $O$, Pootong P, Dalsgaard A, Bodhidatta L, Guerry P, Tribble DR, Anuras S, Mason CJ. PFGE, Lior serotype, and antimicrobial resistance patterns among Campylobacter jejuni isolated from travelers and US military personnel with acute diarrhea in Thailand, 1998-2003. Gut Pathog. 2010;2(1):15.

24. Brown HL, Reuter M, Salt L, Cross KL, Betts RP, van Vliet AH. Chicken juice enhances surface attachment and biofilm formation of Campylobacter jejuni. Appl Environ Microbiol. 2014;80(22):7053-60.

25. Yi K, Rasmussen AW, Gudlavalleti SK, Stephens DS, Stojiljkovic I. Biofilm formation by Neisseria meningitidis. Infect Immun. 2004;72(10):6132-8.

26. Jin H, Zhou R, Kang M, Luo R, Cai X, Chen H. Biofilm formation by field isolates and reference strains of Haemophilus parasuis. Vet Microbiol. 2006;118(1-2):117-23.

27. Dingle KE, Colles FM, Wareing DR, Ure R, Fox AJ, Bolton FE, Bootsma HJ, Willems RJ, Urwin R, Maiden MC. Multilocus sequence typing system for Campylobacter jejuni. J Clin Microbiol. 2001;39(1):14-23.

28. Skarp CPA, Hanninen ML, Rautelin HIK. Campylobacteriosis: the role of poultry meat. Clin Microbiol Infect. 2016;22(2):103-9.

29. Sahin O, Kassem II, Shen Z, Lin J, Rajashekara G, Zhang Q. Campylobacter in poultry: ecology and potential interventions. Avian Dis. 2015;59(2):185-200.

30. Huang JL, Xu HY, Bao GY, Zhou XH, Ji DJ, Zhang G, Liu PH, Jiang F, Pan ZM, Liu XF, et al. Epidemiological surveillance of Campylobacter jejuni in chicken, dairy cattle and diarrhoea patients. Epidemiol Infect. 2009;137(8):1111-20.

31. Ma H, Su Y, Ma L, Li P, Du X, Golz G, Wang S, Lu X. Prevalence and characterization of Campylobacter jejuni isolated from retail chicken in Tianjin. China. J Food Prot. 2017;80(6):1032-40.

32. Wang J, Guo YC, Li N. Prevalence and risk assessment of Campylobacter jejuni in chicken in China. Biomed Environ Sci. 2013;26(4):243-8.

33. Piccirillo A, Giacomelli M, Salata C, Bettanello S, De Canale E, Palu G. Multilocus sequence typing of Campylobacter jejuni and Campylobacter coli from humans and chickens in North-eastern Italy. New Microbiol. 2014;37(4):557-62.

34. Ioannidou V, loannidis A, Magiorkinis E, Bagos P, Nicolaou C, Legakis N, Chatzipanagiotou S. Multilocus sequence typing (and phylogenetic analysis) of Campylobacter jejuni and Campylobacter coli strains isolated from clinical cases in Greece. BMC Res Notes. 2013;6:359.

35. Zhang M, GuY, He L, Ran L, Xia S, Han X, Li H, Zhou H, Cui Z, Zhang J. Molecular typing and antimicrobial susceptibility profiles of Campylobacter jejuni isolates from north China. J Med Microbiol. 2010;59(Pt 10):1171-7.

36. Zhang M, Liu X, Xu X, Gu Y, Tao X, Yang X, Yan G, Zhang J. Molecular subtyping and antimicrobial susceptibilities of Campylobacter coli isolates from diarrheal patients and food-producing animals in China. Foodborne Pathog Dis. 2014;11(8):610-9. 
37. Liu D, Deng F, Gao Y, Yao H, Shen Z, Wu C, Wang Y, Shen J. Dissemination of erm(B) and its associated multidrug-resistance genomic islands in Campylobacter from 2013 to 2015. Vet Microbiol. 2017;204:20-4.

38. Hao H, Yuan Z, Shen Z, Han J, Sahin O, Liu P, Zhang Q. Mutational and transcriptomic changes involved in the development of macrolide resistance in Campylobacter jejuni. Antimicrob Agents Chemother. 2013;57(3):1369-78.

39. Zhu XH, Tian L, Cheng ZJ, Liu WY, Li S, Yu WT, Zhang WQ, Xiang X, Sun ZY. Viral and bacterial etiology of acute diarrhea among children under 5 years of age in Wuhan. China. Chin Med J (Engl). 2016;129(16):1939-44.

40. Chen X, Naren GW, Wu CM, Wang Y, Dai L, Xia LN, Luo PJ, Zhang Q, Shen JZ. Prevalence and antimicrobial resistance of Campylobacter isolates in broilers from China. Vet Microbiol. 2010;144(1-2):133-9.

41. Chu YW, Chu MY, Luey KY, Ngan YW, Tsang KL, Kam KM. Genetic relatedness and quinolone resistance of Campylobacter jejuni strains isolated in 2002 in Hong Kong. J Clin Microbiol. 2004;42(7):3321-3.

42. Wang X, Zhao S, Harbottle H, Tran T, Blickenstaff K, Abbott J, Meng J. Antimicrobial resistance and molecular subtyping of Campylobacter jejuni and Campylobacter coli from retail meats. J Food Prot. 2011;74(4):616-21.

43. Zhang T, Cheng Y, Luo Q, Lu Q, Dong J, Zhang R, Wen G, Wang H, Luo $\mathrm{L}$, Liu G et al. Correlation between gyrA and CmeR Box polymorphism and fluoroquinolone resistance in Campylobacter jejuni isolates in China. Antimicrob Agents Chemother 2017;61(7):e00422-17.

44. Lin J, Martinez A. Effect of efflux pump inhibitors on bile resistance and in vivo colonization of Campylobacter jejuni. J Antimicrob Chemother. 2006;58(5):966-72.

45. Mavri A, Smole Mozina S. Effects of efflux-pump inducers and genetic variation of the multidrug transporter $\mathrm{cmeB}$ in biocide resistance of Campylobacter jejuni and Campylobacter coli. J Med Microbiol. 2013;62(Pt 3):400-11.

46. Su CC, Yin L, Kumar N, Dai L, Radhakrishnan A, Bolla JR, Lei HT, Chou TH, Delmar JA, Rajashankar KR, et al. Structures and transport dynamics of a Campylobacter jejuni multidrug efflux pump. Nat Commun. 2017;8(1):171.
47. Kvist M, Hancock V, Klemm P. Inactivation of efflux pumps abolishes bacterial biofilm formation. Appl Environ Microbiol. 2008;74(23):7376-82.

48. Teh AHT, Lee SM, Dykes GA. Identification of potential Campylobacter jejuni genes involved in biofilm formation by EZ-Tn5 Transposome mutagenesis. BMC Res Notes. 2017;10(1):182.

49. Shen L, Yuan X, Shen W, He N, Wang Y, Lu H, Lu Y. Positive impact of biofilm on reducing the permeation of ampicillin through membrane for membrane bioreactor. Chemosphere. 2014;97:34-9.

50. Li L, Xu Z, Zhou Y, Li T, Sun L, Chen H, Zhou R. Analysis on Actinobacillus pleuropneumoniae LUXS regulated genes reveals pleiotropic roles of LuxS/ Al-2 on biofilm formation, adhesion ability and iron metabolism. Microb Pathog. 2011;50(6):293-302.

51. Abdi-Hachesoo B, Khoshbakht R, Sharifiyazdi H, Tabatabaei M, Hosseinzadeh S, Asasi K. Tetracycline resistance genes in Campylobacter jejuni and C. coli isolated from poultry carcasses. Jundishapur J Microbiol. 2014;7(9):e12129.

52. Kudirkiene E, Cohn MT, Stabler RA, Strong PC, Serniene L, Wren BW, Nielsen EM, Malakauskas M, Brondsted L. Phenotypic and genotypic characterizations of Campylobacter jejuni isolated from the broiler meat production process. Curr Microbiol. 2012;65(4):398-406.

53. de Haan CP, Kivisto R, Hanninen ML. Association of Campylobacter jejuni Cj0859c gene (fspA) variants with different C. jejuni multilocus sequence types. Appl Environ Microbiol. 2010;76(20):6942-3.

54. Flanagan RC, Neal-McKinney JM, Dhillon AS, Miller WG, Konkel ME. Examination of Campylobacter jejuni putative adhesins leads to the identification of a new protein, designated FlpA, required for chicken colonization. Infect Immun. 2009;77(6):2399-407.

55. Pascoe B, Meric G, Murray S, Yahara K, Mageiros L, Bowen R, Jones NH, Jeeves RE, Lappin-Scott HM, Asakura H, et al. Enhanced biofilm formation and multi-host transmission evolve from divergent genetic backgrounds in Campylobacter jejuni. Environ Microbiol. 2015;17(11):4779-89.

\section{Submit your next manuscript to BioMed Central and we will help you at every step:}

- We accept pre-submission inquiries

- Our selector tool helps you to find the most relevant journal

- We provide round the clock customer support

- Convenient online submission

- Thorough peer review

- Inclusion in PubMed and all major indexing services

- Maximum visibility for your research

Submit your manuscript at www.biomedcentral.com/submit
() Biomed Central 